\title{
Dielectric behavior and phase transition in [111]-oriented PIN-PMN-PT single crystals under de bias
}

\author{
Yuhui Wan*, ${ }^{*}$, Zhenrong Li*, ${ }^{*}$, Ming Ma*, Fei Li*, \\ Zhuo Xu*, Shiji Fan* and Xi Yao* \\ *Electronic Materials Research Laboratory, Key Laboratory of the \\ Ministry of Education \& International Center for Dielectric Research, Xi'an \\ Jiaotong University, Xi'an 710049, P. R. China \\ ${ }^{\dagger}$ School of Materials Science and Engineering \\ $X i$ 'an University of Technology, Xi'an 710048, P. R. China \\ tzhrli@mail.xjtu.edu.cn
}

Received 26 December 2013; Revised 25 February 2014; Accepted 3 March 2014; Published 27 March 2014

\begin{abstract}
Temperature and electric field dependences of the dielectric behavior and phase transition for [111]-oriented 0.23PIN-0.52PMN0.25PT (PIN-PMN-0.25PT) and 0.24PIN-0.43PMN-0.33PT (PIN-PMN-0.33PT) single crystals were investigated over a temperature range from $-100^{\circ} \mathrm{C}$ to $250^{\circ} \mathrm{C}$ using field-heating $(\mathrm{FH})$ dielectric measurements. The transition phenomenon from ferroelectric microdomain to macrodomain was found in rhombohedra $(\mathrm{R})$ phase region in the single crystals under dc bias. This transition temperature $T_{f}$ of micro-to-macrodomain is sensitive to dc bias and move quickly to lower temperature with increasing dc bias. The phase transition temperatures in the two single crystals shift toward high temperature and the dielectric permittivities at the phase transition temperature decrease with increasing dc bias. Especially, the phase transition peaks are gradually broad in PINPMN-0.33PT single crystal with the increasing dc bias. Effects of dc bias on the dielectric behavior and phase transition in PINPMN-PT single crystals are discussed.
\end{abstract}

Keywords: PIN-PMN-PT single crystal; electric field; phase transition; dielectric.

\section{Introduction}

The relaxor-based single crystal $\mathrm{Pb}\left(\mathrm{In}_{1 / 2} \mathrm{Nb}_{1 / 2}\right) \mathrm{O}_{3}-\mathrm{Pb}$ $\left(\mathrm{Mg}_{1 / 3} \mathrm{Nb}_{2 / 3}\right) \mathrm{O}_{3}-\mathrm{PbTiO}_{3}$ (PIN-PMN-PT) with morphotropic phase boundary (MPB) composition have excellent piezoelectric properties of $d_{33} \sim 2000 \mathrm{pC} / \mathrm{N}$ and $k_{33} \sim 95 \%$, meanwhile the depoling temperature is higher $20-30^{\circ} \mathrm{C}$ than that of binary $\mathrm{Pb}\left(\mathrm{Mg}_{1 / 3} \mathrm{Nb}_{2 / 3}\right) \mathrm{O}_{3}-\mathrm{PbTiO}_{3}$ (PMN-PT) single crystal. Due to these merits, many studies have been carried out on the relationship between piezoelectric properties and MPB compositions. ${ }^{1-3}$ For PMN-PT single crystals, the dielectric properties and phase transition characteristics under dc bias were systematically studied. ${ }^{4-7}$ It was found that the appearance/stability of the intermediate phases is strongly dependent on the direction and strength of the electric field and the orientation of the crystal. ${ }^{4}$ For PMN-PT single crystal with MPB composition, the intermediate $\mathrm{M}$ and $\mathrm{O}$ phases can be induced during the field heating process for both the [110]- and [001]-oriented crystals but not for the [111]-oriented crystals. For $0.76 \mathrm{PMN}-0.24 \mathrm{PT}$ single crystal with composition far away from MPB, the diffusion index under dc bias along [001], [011] and [111] direction was closely related with the value, not the direction, of dc bias. ${ }^{8}$ Comparing to PMN-PT single crystals, the dielectric properties and phase transition characteristics of PIN-PMN-PT single crystal under dc bias will possibly be more complicated than that in PMN-PT single crystals due to multiple B site ions.

Owing to the inevitable compositional segregation in the PIN-PMN-PT crystal growth process, the composition along the crystal growth direction is also different, ${ }^{9}$ resulting in variation of dielectric properties and phase transition characteristics. In this paper, the dielectric behavior and phase transition characteristics of [111]-oriented 0.23PIN0.52PMN-0.25PT (PIN-PMN-0.25PT, R phase composition) and 0.24PIN-0.43PMN-0.33PT (PIN-PMN-0.33PT, MPB composition) single crystals under different electric fields along [111] direction were characterized over a broad temperature range from $-100^{\circ} \mathrm{C}$ to $250^{\circ} \mathrm{C}$. The microdomain to macrodomain transition was found, which would lead to a good understanding of the dielectric behavior of the ternary single crystal.

\section{Experimental Procedure}

PIN-PMN-PT single crystal was grown by a modified Bridgman method. The composition of the single crystals were analyzed by an electron probe X-ray microanalyzer (EPMA, JEOL JXA-8230 superprobe). ${ }^{10}$ [111]-oriented PIN-PMN-0.25PT and PIN-PMN-0.33PT single crystals of

This is an Open Access article published by World Scientific Publishing Company. It is distributed under the terms of the Creative Commons Attribution 3.0 (CC-BY) License. Further distribution of this work is permitted, provided the original work is properly cited. 
size of $5 \times 5 \times 0.5 \mathrm{~mm}^{3}$ were chosen for dielectric measurements. Silver electrodes were fired on both faces of the samples at $600^{\circ} \mathrm{C}$ for $25 \mathrm{~min}$. Dielectric measurements were performed at frequencies of $0.1,1,10$ and $100 \mathrm{kHz}$, respectively, over a temperature range from $-100^{\circ} \mathrm{C}$ to $250^{\circ} \mathrm{C}$ with a heating rate of $2^{\circ} \mathrm{C} \cdot \min$ by using an automated system where a computer was employed to control a Delta temperature box, an HP4284A LCR meter, and a high voltage generator (Model PS350/5000V-25W, Stanford research system, INC.). The dielectric properties of the samples were measured using field-heating $(\mathrm{FH})$ process, in which the sample was heated under a constant dc bias along [111] direction. The $\mathrm{dc}$ bias was from 0 to $5 \mathrm{kV} \cdot \mathrm{cm}^{-1}$. To eliminate the influence of the sample history on the results, all the samples were annealed at $400^{\circ} \mathrm{C}$ for $2 \mathrm{~h}$ prior to the dielectric measurements.

\section{Results and Discussion}

Temperature dependence of dielectric permittivity $\left(\varepsilon^{\prime}\right)$ at 0.1 , 1,10 , and $100 \mathrm{kHz}$ and $\operatorname{loss}(\tan \delta)$ at $1 \mathrm{kHz}$ for [111]-oriented PIN-PMN-0.25PT single crystal under dc bias of 0, 1, 2, 3 and $4 \mathrm{kV} \cdot \mathrm{cm}^{-1}$ is shown in Figs. 1(a)-1(e), respectively. As shown in Fig. 1(a), without dc bias, the obvious dielectric permittivity frequency dependence can be seen near $T_{m} \sim 150^{\circ} \mathrm{C}$ (peak I) with $\Delta T_{\text {dispersion }}\left(=T_{m, 100 \mathrm{kHz}}-T_{m, 0.1 \mathrm{kHz}}\right)=8.4^{\circ} \mathrm{C}$. The $\tan \delta$ is found to remain substantially unchanged with a value of 0.06 in the range from room temperature to $110^{\circ} \mathrm{C}$, and increases to a maximum value of 0.09 near $135^{\circ} \mathrm{C}$, which is determined to be the phase transition of rhombohedral $(\mathrm{R})$ to Cubic (C) phase. ${ }^{11} \mathrm{It}$ is noted that the loss peaks around $0{ }^{\circ} \mathrm{C}$ for all samples are related to water vapor during the measurement process.

Under dc bias of $1 \mathrm{kV} \cdot \mathrm{cm}^{-1}$, the peak labeled $T_{f}$ observed near $50^{\circ} \mathrm{C}$ is found, which is a new phenomenon heretofore unobserved in PIN-PMN-PT system. Below $T_{f}$, the dielectric permittivity frequency dispersion is shown in inset of Fig. 1(b). When the temperature is once over $T_{f}, \varepsilon^{\prime}$ and $\tan \delta$ have clear step-like decrease from $\sim 2450$ to $\sim 900$ and from $\sim 0.063$ to $\sim 0.016$ at $1 \mathrm{kHz}$, respectively, and the frequency dispersion of $\varepsilon^{\prime}$ almost disappears, as shown in Fig. 1(b). By increasing the temperature further, a sharp dielectric peak A can be clearly seen near $125^{\circ} \mathrm{C}$, and the temperature corresponding to which is nearly independent of frequency and the dielectric peak values decrease with increasing frequency. The dielectric permittivity frequency dispersion near $T_{m} \sim 150^{\circ} \mathrm{C}$ (peak I) is also evident with $\Delta T_{\text {dispersion }}=8.3^{\circ} \mathrm{C}$ and disappear above $\sim 160^{\circ} \mathrm{C}$. The dielectric permittivity peak actually reflects two processes: one is the phase transition R-to-C (peak A) and the other is the ferroelectric relaxor process (peak I). It means that the phase transition and relaxor process could be split under small de bias.
The dielectric anomaly at temperature $T_{f}$ is assumed to be the micro-macro domain transition. ${ }^{12}$ When the temperature is below $T_{f}$, the polar region and part of microdomains with arbitrary polarization direction exist. The thermal energy is not sufficient to overcome the barrier of dipole reversion, resulting in evident dielectric dispersion. Thermal energy overcomes the barrier of dipole reversion when the temperature is above $T_{f}$. So the dipoles in microdomains are arranged along the direction of dc bias resulting in the rapid reduction of dielectric dispersion and dielectric loss in Fig. 1(b).

Similarly to $1 \mathrm{kV} \cdot \mathrm{cm}^{-1}, T_{f}$ is also observed around $0^{\circ} \mathrm{C}$, $-30^{\circ} \mathrm{C},-50^{\circ} \mathrm{C}$ when dc bias is 2,3 and $4 \mathrm{kV} \cdot \mathrm{cm}^{-1}$, respectively, as shown in Figs. 1(c)-1(e). With the increase of dc bias, $T_{f}$ clearly moves to lower temperature. It can also be found that $T_{f}$ is sensitive to dc bias. When $\mathrm{dc}$ bias is increased, the dynamics of micro-domains reconstruction is accelerated and the thermal energy needed to overcome the barriers of dipole's reversion in microdomain decreases. Therefore, the transition temperature of microdomain to macrodomain moves to lower temperature.

Compared to Fig. 1(b), the dielectric peak A is found to disappear or move to higher temperature and merge together with $T_{m}$ under dc bias of $2 \mathrm{kV} \cdot \mathrm{cm}^{-1}$, as shown in Fig. 1(c). The dielectric dispersion at $T_{m}$ is also evident with $\Delta T_{\text {dispersion }}=11.8^{\circ} \mathrm{C}$. With the further increase of dc bias, dielectric dispersion around $T_{m}$ decreases greatly with $\Delta T_{\text {dispersion }}=3.4^{\circ} \mathrm{C}$ and $1.8^{\circ} \mathrm{C}$ when dc bias increases to $3 \mathrm{kV} \cdot \mathrm{cm}^{-1}$ (Fig. 1(d)) and $4 \mathrm{kV} \cdot \mathrm{cm}^{-1}$ (Fig. 1(e)). Dielectric dispersion $\Delta T_{\text {dispersion }}$ also decreases with increase of dc bias for enhancement of orientation of dc bias on macrodomain.

Comparing to PIN-PMN-0.25PT single crystal with $\mathrm{R}$ phase, the $\varepsilon^{\prime}-T$ curve under dc bias for PIN-PMN-0.33PT single crystal with MPB composition is more complicated, as seen in Fig. 2. Only one dielectric peak (peak I) near $194^{\circ} \mathrm{C}$ is found with weak dielectric permittivity frequency dispersion of $\Delta T_{\text {dispersion }}=0.9^{\circ} \mathrm{C}$ under $0 \mathrm{kV} \cdot \mathrm{cm}^{-1} \mathrm{dc}$ bias, as shown in Fig. 2(a). The dielectric permittivity frequency dispersion of PIN-PMN-PT single crystal reduces with the increase of PT content, comparing to Figs. 1(a) and 2(a). As dc bias increases to $1 \mathrm{kV} \cdot \mathrm{cm}^{-1}$ (Fig. 2(b)), Peak I splits into two peaks and the corresponding temperatures are $186^{\circ} \mathrm{C}$ and $191^{\circ} \mathrm{C}$, respectively. The dielectric peak around $186^{\circ} \mathrm{C}$ is found to be sharper than the peak around $191^{\circ} \mathrm{C}$. The shape of dielectric peak I is similar to the " $\lambda$-like" dielectricpermittivity peak in [111]-oriented 0.68PMN-0.32PT single crystal under electric fields. ${ }^{4} \mathrm{~A}$ peak at $186^{\circ} \mathrm{C}$ is corresponding to the transition of tetragonal $(\mathrm{T})$ phase to Cubic $(\mathrm{C})$ phase. The peak position is independent of frequency, and the peak value decreases with increase of frequency. Another peak at $191^{\circ} \mathrm{C}$ is corresponding to the ferroelectric relaxor process with $\Delta T_{\text {dispersion }}=1.7^{\circ} \mathrm{C}$. On the lower temperature side of peak I, peak II at $130^{\circ} \mathrm{C}$ is found to be related to R-to-T phase transition. The dielectric loss double peaks corresponding to 


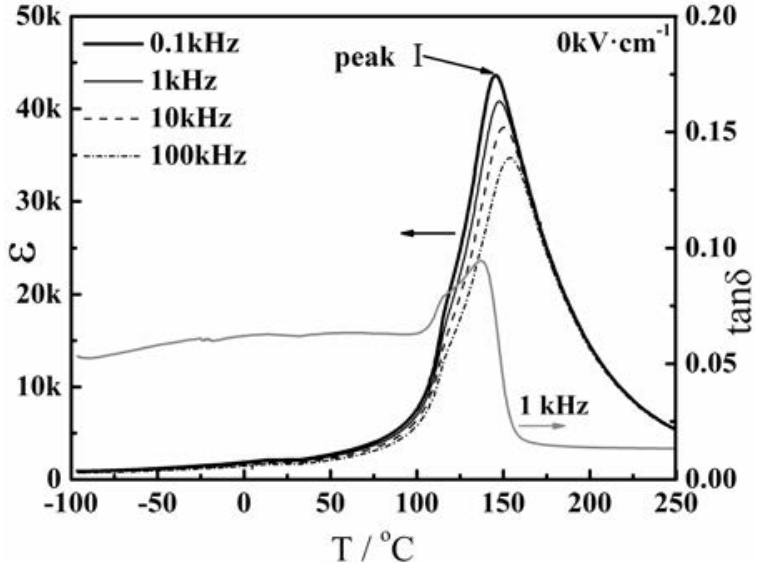

(a)

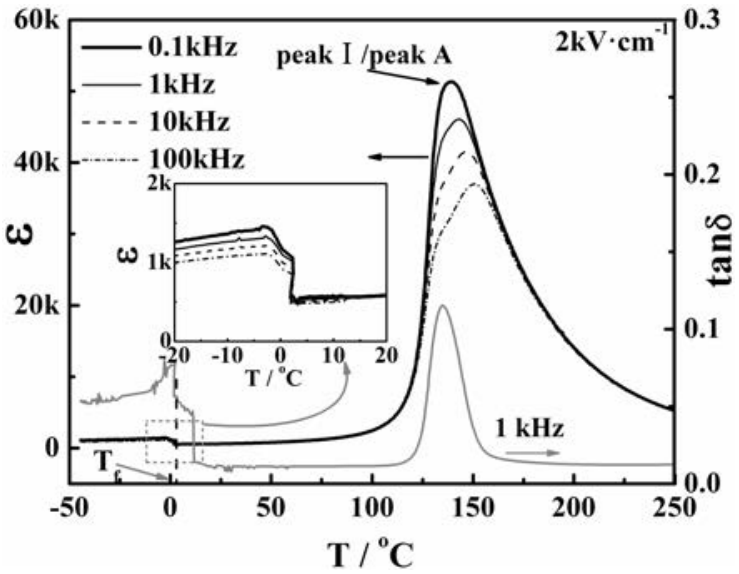

(c)

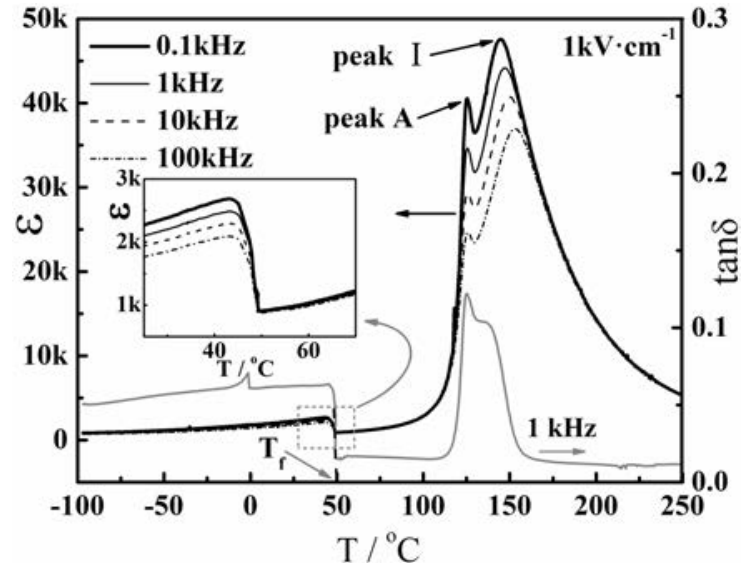

(b)

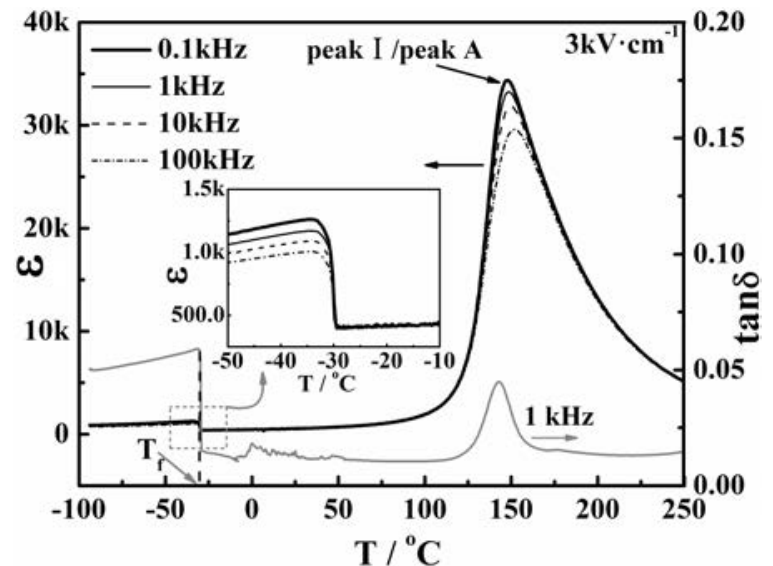

(d)

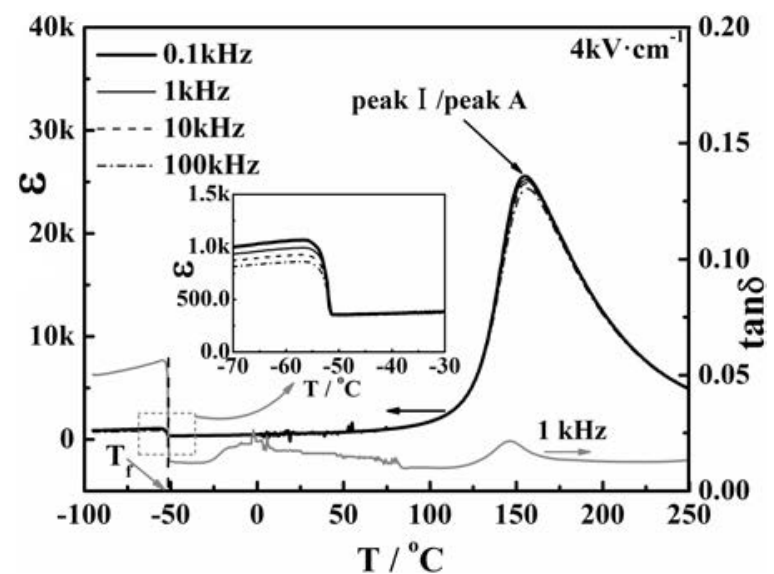

(e)

Fig. 1. Temperature dependence of dielectric permittivity at $0.1,1,10$ and $100 \mathrm{kHz}$ and loss at $1 \mathrm{kHz}$ for PIN-PMN-0.25PT single crystal during FH with different dc biases: (a) $0 \mathrm{kV} \cdot \mathrm{cm}^{1}$, (b) $1 \mathrm{kV} \cdot \mathrm{cm}^{1}$, (c) $2 \mathrm{kV} \cdot \mathrm{cm}^{1}$, (d) $3 \mathrm{kV} \cdot \mathrm{cm}^{1}$ and (e) $4 \mathrm{kV} \cdot \mathrm{cm}^{1}$.

peak II can be clearly seen, and the loss peak on the lower temperature side may be related to the phase transition of $\mathrm{R}$ to $\mathrm{O}$, the loss peak on the higher temperature side may be related to the phase transition of $\mathrm{O}$ to $\mathrm{T}^{13}$ Therefore, the phase transition sequence for [111]-oriented PIN-PMN-0.33PT single crystal under dc bias during heating process may be $\mathrm{R} \rightarrow \mathrm{O} \rightarrow \mathrm{T} \rightarrow \mathrm{C}$. However, the temperature region of $\mathrm{O}$ phase is very narrow. 


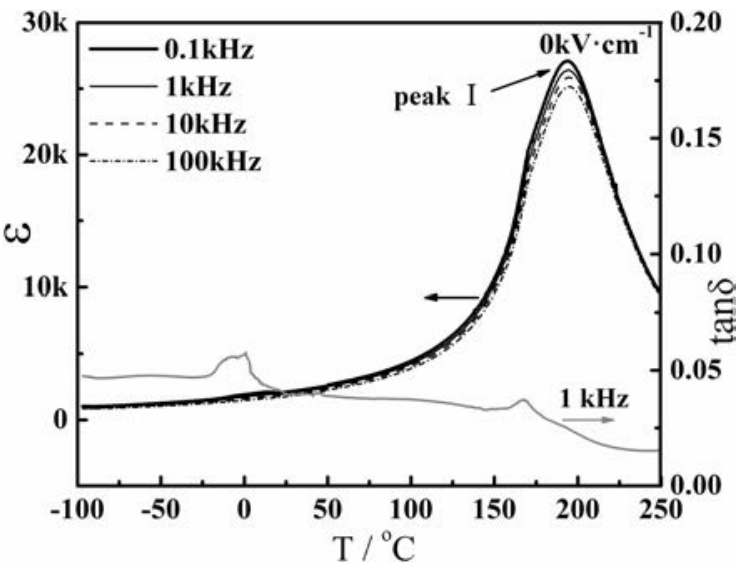

(a)

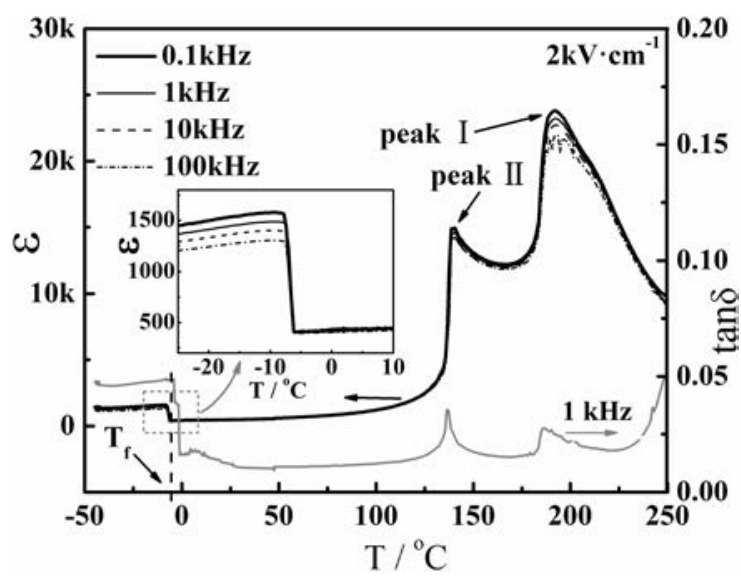

(c)

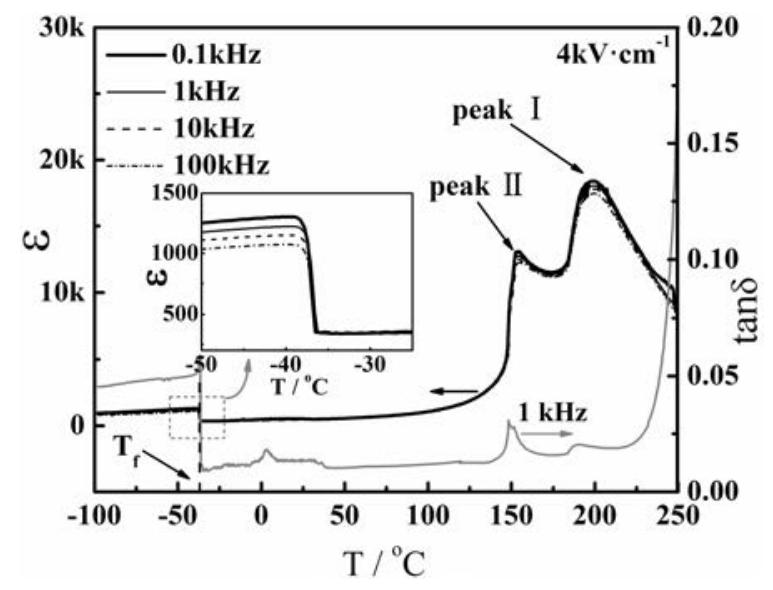

(e)

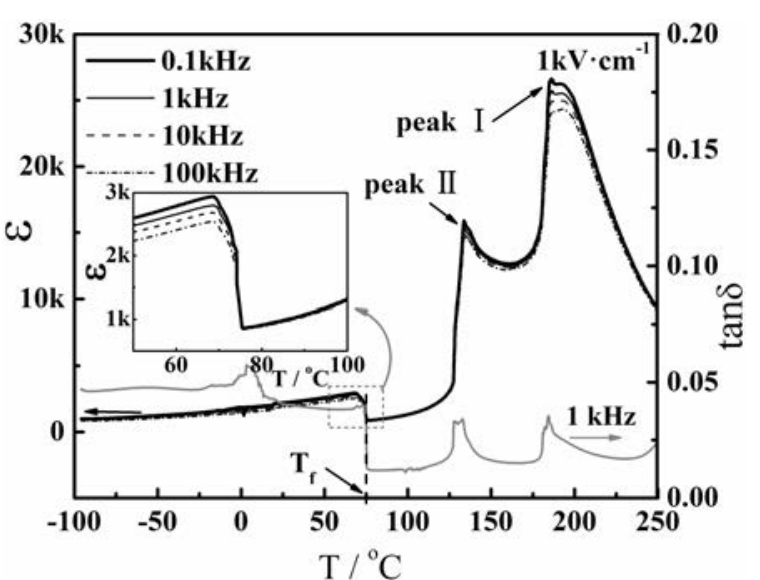

(b)

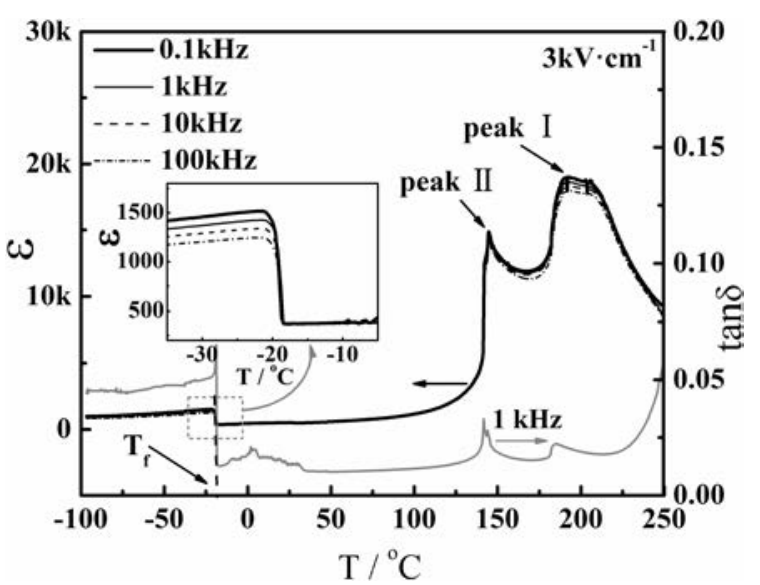

(d)

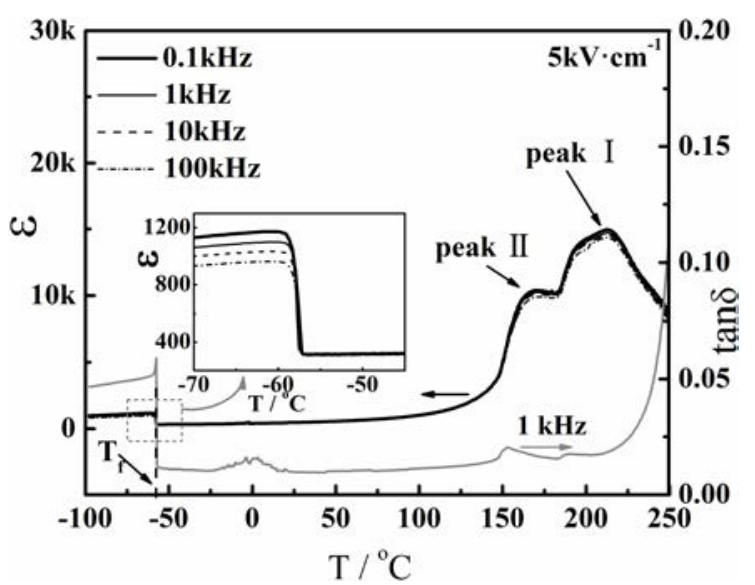

(f)

Fig. 2. Temperature dependence of dielectric permittivity at $0.1,1,10$ and $100 \mathrm{kHz}$ and loss at $1 \mathrm{kHz}$ for PIN-PMN-0.33PT single crystal during FH with different dc biases: (a) $0 \mathrm{kV} \cdot \mathrm{cm}^{1}$, (b) $1 \mathrm{kV} \cdot \mathrm{cm}^{1}$, (c) $2 \mathrm{kV} \cdot \mathrm{cm}^{1}$, (d) $3 \mathrm{kV} \cdot \mathrm{cm}^{1}$, (e) $4 \mathrm{kV} \cdot \mathrm{cm}^{1}$ and (f) $5 \mathrm{kV} \cdot \mathrm{cm}^{1}$.

An interesting phenomenon similar to Fig. 1 is found around the temperature of $70^{\circ} \mathrm{C}\left(T_{f}\right)$, below which dielectric permittivity is dependent on frequency, and above which dielectric permittivity is nearly independent of frequency, as can be clearly seen in the insets of Fig. 2(b). When the temperature increases over $T_{f} \sim 70^{\circ} \mathrm{C}$, dielectric permittivity and loss at $1 \mathrm{kHz}$ decrease quickly from $\sim 2938$ to $\sim 868$ and from $\sim 0.040$ to $\sim 0.012$, respectively. Under dc bias of 


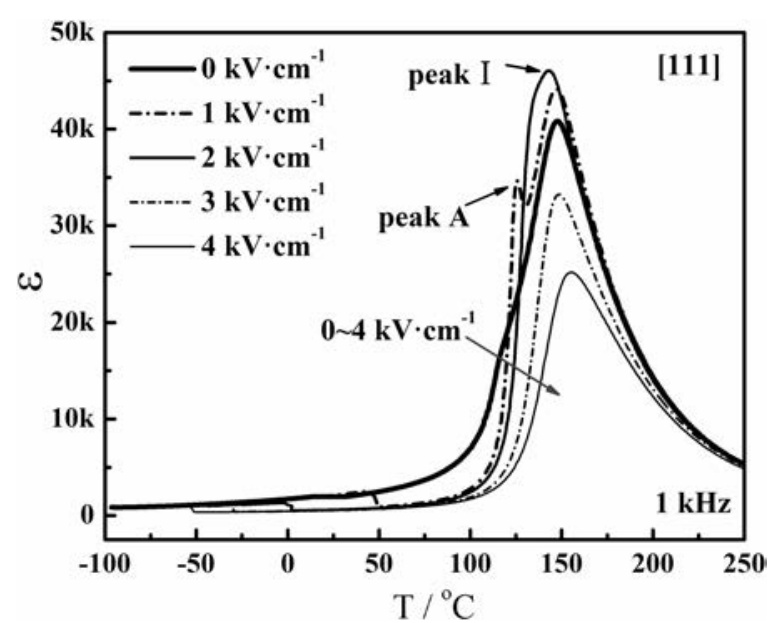

(a)

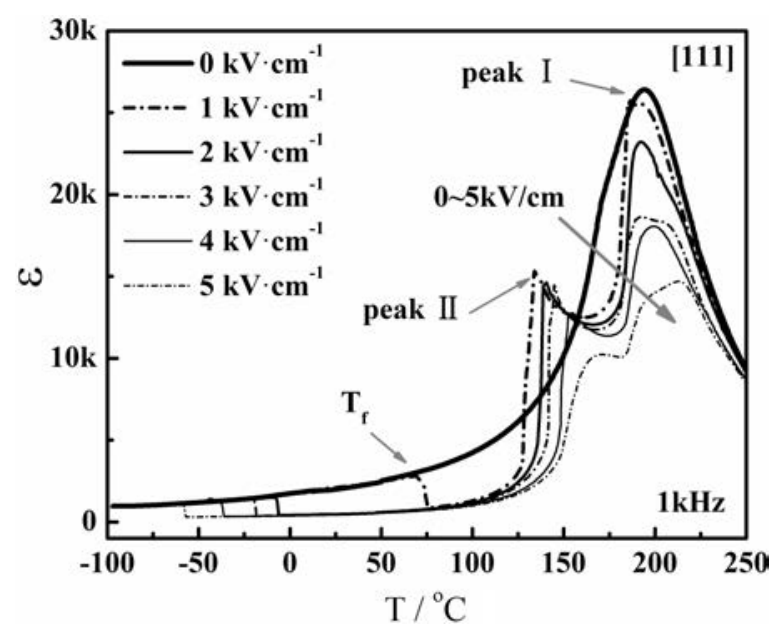

(b)

Fig. 3. Temperature dependence of dielectric permittivity at $1 \mathrm{kHz}$ during heating process under dc biases for [111] single crystal with different compositions: (a) PIN-PMN-0.25PT and (b) PIN-PMN$0.33 \mathrm{PT}$.

$1 \mathrm{kV} \cdot \mathrm{cm}^{-1}$, the change of dielectric properties around $70^{\circ} \mathrm{C}$ is similar to that around $50^{\circ} \mathrm{C}$ for PIN-PMN-0.25PT single crystal.

With the increase of dc bias, peak I and peak II move to higher temperature, while $T_{f}$ moves to lower temperature, as can be seen in Figs. 2(c)-2(f). When dc bias increases to $5 \mathrm{kV} \cdot \mathrm{cm}^{-1}$, peak II and peak II are broad, and move to $212.7^{\circ} \mathrm{C}$ and $166.3^{\circ} \mathrm{C}$, with dielectric permittivity of 14,700 and 10,200 , respectively, along with $T_{f}$ moving to $-57^{\circ} \mathrm{C}$.

Another interesting phenomenon can be found in dielectric loss corresponding to peak II under dc bias of $2 \mathrm{kV} \cdot \mathrm{cm}^{-1}$ with only one peak in Fig. 2(c), unlike the double loss peaks in Fig. 2(b). The double loss peaks corresponding to peak II reappear when the dc bias further increases to $3 \mathrm{kV} \cdot \mathrm{cm}^{-1}$, and disappear under $5 \mathrm{kV} \cdot \mathrm{cm}^{-1}$. It can be known that the loss peak on the lower temperature side may be related to the phase transition of $\mathrm{R}$ to $\mathrm{O}$, the loss peak on the higher temperature side may be related to the phase transition of $\mathrm{O}$ to $\mathrm{T}$. Therefore, it can be speculated that the dielectric loss peaks related to peak II is sensitive to dc bias, that is, $\mathrm{O}$ phase is unstable and sensitive to $\mathrm{dc}$ bias in [111]-oriented PIN-PMN-PT single crystal.

Temperature dependence of dielectric permittivity at $1 \mathrm{kHz}$ under different dc bias for PIN-PMN-0.25PT and PIN-PMN-0.33PT single crystals is shown in Figs. 3(a) and 3 (b), respectively. It can be seen that the $\varepsilon^{\prime}-\mathrm{T}$ curve for PINPMN-0.33PT single crystal in Fig. 3(b) is more complicated than that for PIN-PMN-0.25PT single crystal in Fig. 3(a). Dielectric peak II in Fig. 3(b), related to phase transition of R to $\mathrm{T}$, is found to move to higher temperature with the increase of dc bias. For PIN-PMN-0.33PT existed in the MPB of PIN-PMN-PT system, determined from previous works, ${ }^{10}$ multiphase coexist with nearly the same free energy and the $\mathrm{T}$ phase can be easily induced by combined effect of dc bias and temperature. For 24PIN-PMN-32PT single crystals with MPB compositions, dc bias can also stabilize the $\mathrm{R}$ phase under large signal stress-strain. ${ }^{14}$ As PIN-PMN-0.33PT single crystal at low temperature belongs to rhombohedral phase with spontaneous polarization [111] direction, dc bias applied along [111] direction can increase the $\mathrm{R}$ phase stability, and then expand the temperature range where $\mathrm{R}$ phase exists, making the phase transition temperature of $\mathrm{R}$ to $\mathrm{T}$ move to higher temperature.

The transition temperature $T_{f}$ of ferroelectric microdomain to macrodomain for [111]-oriented PIN-PMN-PT single crystal moves to lower temperature with the increase of $\mathrm{dc}$ bias whether the PT content is low or high, as shown in Fig. 4. It is also found that $T_{f}$ for [111]-oriented PIN-PMN0.33PT single crystal is higher than that for PIN-PMN$0.25 \mathrm{PT}$ single crystal under the same dc bias, except $2 \mathrm{kV} \cdot \mathrm{cm}^{-1}$. We carried out the same dielectric measurements on [111]-oriented PMN-0.32PT single crystal. But $T_{f}$ was not found. ${ }^{4}$ Although the reason for $T_{f}$ is not clear, $T_{f}$ depends on the composition of the single crystal and the strength of the electric field.

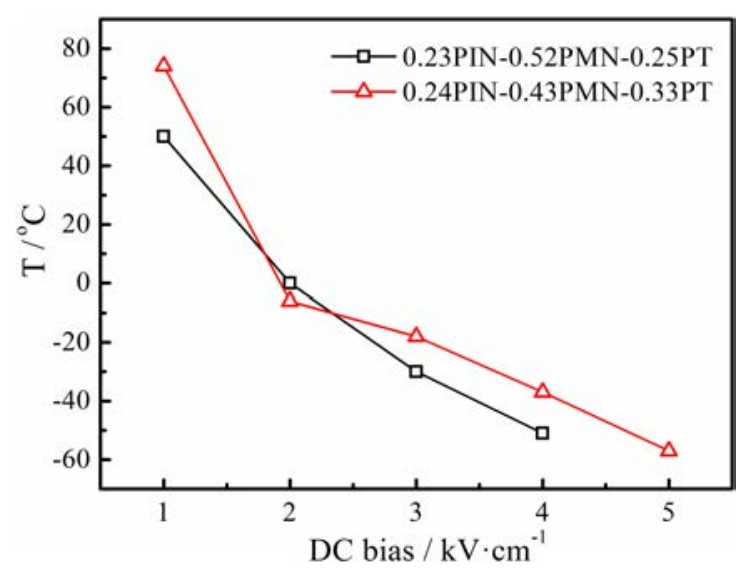

Fig. 4. $T_{f}$ as a function of dc bias for [111]-oriented PIN-PMN-PT single. 


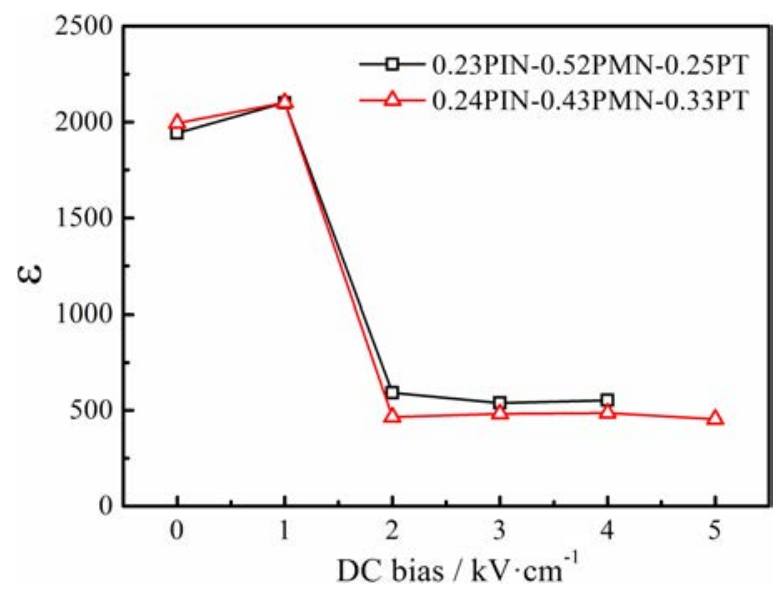

Fig. 5. The dc bias dependence of the dielectric permittivity at $1 \mathrm{kHz}$ for [111]-oriented crystals at room temperature.

The dc bias dependence of the dielectric permittivity at $1 \mathrm{kHz}$ for [111]-oriented crystals with different compositions at room temperature are depicted in Fig. 5. It is found that dielectric permittivity of these two crystals under $0 \mathrm{kV} \cdot \mathrm{cm}^{-1}$ are nearly same with the value of $\sim 2000$. When dc bias increase to $1 \mathrm{kV} \cdot \mathrm{cm}^{-1}$, dielectric permittivity increase slightly to $\sim 2100$ and then decrease quickly to $\sim 500$ under $\mathrm{dc}$ bias of $2 \mathrm{kV} \cdot \mathrm{cm}^{-1}$. When the dc bias is more than $2 \mathrm{kV} \cdot \mathrm{cm}^{-1}$, the dielectric permittivity is essentially unchanged. It is well know that the dielectric permittivity of PIN-PMN-PT crystal along the spontaneous polarization [111] direction is less than that along other direction. Part of the ferroelectric domains is arranged along the direction of $\mathrm{dc}$ bias when dc is was not so high enough. As dc bias along [111] direction is over $2 \mathrm{kV} \cdot \mathrm{cm}^{-1}$, ferroelectric domains are arranged almost along electric field, resulting in the significant reduction of dielectric permittivity.

\section{Conclusion}

The dielectric behavior and phase transition induced by electric field and temperature for [111]-oriented PIN-PMN0.25PT and PIN-PMN-0.33PT single crystals were investigated. The transition temperatures $T_{f}$ of ferroelectric microdomain to macrodomain in $\mathrm{R}$ phase region is found. $T_{f}$ depends on the composition of the single crystal and the strength of the electric field. For [111]-oriented PIN-PMN$0.25 \mathrm{PT}$ single crystal, the phase transition temperature of Rto-C increases and the dielectric permittivity decreases at phase transition peaks with increasing dc bias. In PIN-PMN$0.33 \mathrm{PT}$ single crystal, $\mathrm{T}$ phase and $\mathrm{O}$ phase can be induced by combined effect of dc bias and temperature. The $\mathrm{O}$ phase is unstable and sensitive to dc bias. The phase transition path is $\mathrm{R} \rightarrow($ or $\mathrm{O}) \rightarrow \mathrm{T} \rightarrow \mathrm{C}$. The phase transition temperatures move to high temperature and the phase transition peaks of T-to-C were clearly broad with the increasing dc bias.

\section{Acknowledgments}

This work was financially supported by the National High Technology Research and Development Program of China (863 Program) (Grant No. 2012AA03A704).

\section{References}

${ }^{1} \mathrm{~S}$. Zhang, J. Luo, W. Hackenberger et al., Electromechanical characterization of $\mathrm{Pb}\left(\mathrm{In}_{0.5} \mathrm{Nb}_{0.5}\right) \mathrm{O}_{3}-\mathrm{Pb}\left(\mathrm{Mg}_{1 / 3} \mathrm{Nb}_{2 / 3}\right) \mathrm{O}_{3}-\mathrm{PbTiO}_{3}$ crystals as a function of crystallographic orientation and temperature, J. Appl. Phys. 105(10), 104506 (2009).

${ }^{2}$ J. Tian, P. Han, X. Huang et al., Improved stability for piezoelectric crystals grown in the lead indium niobate-lead magnesium niobate-lead titanate system, Appl. Phys. Lett. 91(22), 222903 (2007).

${ }^{3}$ S. Zhang, F. Li, N. P. Sherlock et al. Recent Developments on High Curie Temperature PIN-PMN-PT Ferroelectric Crystals, J. Cryst. Growth. 318(1), 846 (2011).

${ }^{4} \mathrm{Z}$. Li, Z. Xu, X. Yao et al., Phase transition and phase stability in [110]-,[001]-, and [111]-oriented $0.68 \mathrm{~Pb}\left(\mathrm{Mg}_{1 / 3} \mathrm{Nb}_{2 / 3}\right) \mathrm{O}_{3}-0.32$ $\mathrm{PbTiO}_{3}$ single crystal under electric field, J. Appl. Phys. 104(2), 024112 (2008).

${ }^{5}$ E. V. Colla, N. K. Yushin and D. Viehland, Dielectric properties of $(\mathrm{PMN})_{(1-x)}(\mathrm{PT})_{x}$ single crystals for various electrical and thermal histories, J. Appl. Phys. 83(6), 3298 (1998).

${ }^{6}$ C.-S. Tu, C. L. Tsai, J. S. Chen et al., Orientation dependence and electric-field effect in the relaxor-based ferroelectric crystal $\left(\mathrm{PbMg}_{1 / 3} \mathrm{Nb}_{2 / 3} \mathrm{O}_{3}\right)_{0.68}\left(\mathrm{PbTiO}_{3}\right)_{0.32}$, Phys. Rev. B 65(10), 104113 (2002).

${ }^{7}$ Y. Guo, H. Luo, D. Ling et al., The phase transition sequence and the location of the morphotropic phase boundary region in $(1-\mathrm{x})$ $\left[\mathrm{Pb}\left(\mathrm{Mg}_{1 / 3} \mathrm{Nb}_{2 / 3}\right) \mathrm{O}_{3}\right]-\mathrm{xPbTiO}_{3}$ single crystal, J. Phys: Condens. Matter 15(2), L77 (2003).

${ }^{8} \mathrm{~S}$. Lu, Z. Xu, H. Chen et al., Effect of dc bias on the Curie-Weiss exponent in $0.76 \mathrm{~Pb}\left(\mathrm{Mg}_{1 / 3} \mathrm{Nb}_{2 / 3}\right) \mathrm{O}_{3}-0.24 \mathrm{PbTiO}_{3}$ ferroelectric single crystal, Appl. Phys. Lett. 86(14), 142905 (2005).

${ }^{9} \mathrm{~S}$. Zhang and F. Li, High performance ferroelectric relaxor$\mathrm{PbTiO}_{3}$ single crystals: Status and perspective, J. Appl. Phys. 111(3), 031301 (2012).

${ }^{10} \mathrm{Y}$. Wan, Z. Li, H. Chen et al. Variations of composition and dielectric properties of $\mathrm{Pb}\left(\mathrm{In}_{1 / 2} \mathrm{Nb}_{1 / 2}\right) \mathrm{O}_{3}-\mathrm{Pb}\left(\mathrm{Mg}_{1 / 3} \mathrm{Nb}_{2 / 3}\right) \mathrm{O}_{3}$ $\mathrm{PbTiO}_{3}$ single crystal along growth direction, J. Appl. Phys. 113(12), 124105 (2013).

${ }^{11}$ Y. Wan, Z. Li, Z. Xu et al., Phase transition characteristics of the relaxor-based 0.24 PIN-0.51 PMN-0.25 PT single crystals, J. Alloy Compd. 558, 244 (2013).

${ }^{12}$ Z. Cheng, L. Zhang and X. Yao, Effect of space charge on micromacro domain transition of PLZT, IEEE Trans. Electr. Insul. 27(4), 773 (1992).

${ }^{13}$ M. Amin Hentati, H. Dammak, H. Khemakhem et al., Dielectric properties and phase transitions of [001],[110], and [111] oriented $\mathrm{Pb}\left(\mathrm{Zn}_{1 / 3} \mathrm{Nb}_{2 / 3}\right) \mathrm{O}_{3}-6 \% \mathrm{PbTiO}_{3}$ single crystals, J. Appl. Phys. 113(24), 244104 (2013).

${ }^{14}$ P. Finkel, H. Robinson, J. Stace et al., Study of phase transitions in ternary lead indium niobate-lead magnesium niobate-lead titanate relaxor ferroelectric morphotropic single crystals, Appl. Phys. Lett. 97(12), 122903 (2010). 